\title{
Application of the Proposed e-CBT Framework in the Development of e-Competency-based Training Web App
}

\author{
Alain C. Gallarde ${ }^{1}$, Irma T. Plata ${ }^{2}$ \\ ${ }^{1}$ Southern Isabela College of Arts and Trades, Santiago City, Philippines \\ acgallarde@tesda.gov.ph \\ ${ }^{2}$ College of Computing Studies, Information and Communication Technology \\ Isabela State University, Echague, Isabela, Philippines \\ irma.t.plata@isu.edu.ph
}

\begin{abstract}
Competency-Based Training (CBT) is a training delivery approach that focuses on the competency development of trainees as a result of training. The proposed e-Competency Based Training (e-CBT) framework was designed and applied in the development of the e-Competency-based training web App for TESDA Technology Institutions with the end-goal of improving the overall results of the competency-based training in learning Computer Systems Servicing (CSS) program/qualification. It further offers the advantage of real-time progress monitoring and automates the majority of tasks like the recording of self-checks and tests. The proposed e-CBT framework covers five significant activities, the pre-assessment, selection of competencies, the learning module, institutional assessment, and the achievement chart. The Rapid Application Development (RAD) model serves as the software development methodology. The Courselab e-learning authoring tool is utilized in the development of the e-CBT web App. The conduct of usability evaluation and acceptability evaluation adopting Jakob Nielsen and the Kirkpatrick evaluation model/tool. Overall, the technical experts highly commended the App in terms of usability. More, the high acceptability rating reveals that the usage of the App provides a more convenient and productive approach in learning. As a result, the proposed framework aids in developing the e-CBT programs/qualification addressing the need for adopting an e-learning system to sustain TESDA's Medium-Term development plan. The developed e-CBT serves as an avenue for trainees to improve their knowledge, skills, and attitude, combining both the self-learning approach and formal classroom instruction. Thus, the web App as an education delivery medium provides current, organize, and accurate information and improves the competency of trainees extending learning outside classroom and time boundaries.
\end{abstract}

Key words: Competency-based training, Courselab. e-CBT, e-Learning, TESDA, Web App

\section{INTRODUCTION}

\subsection{Web-based Learning (WBL)}

Web-based learning (WBL) from a technological point of view is closely related to $e$-learning, internet-based learning, online learning, and computer-based learning concepts. It includes both network-based learning and non-network-based learning. Web-based learning uses Information and Communication Technology (ICT) tools to deliver information for education purposes where the separation of trainers and trainees by distance, time, or both to enhance the trainees' learning experience and performance [1], [2]. Educational institutions are capitalizing on web-based learning systems/platforms supporting modern teaching practices and improving trainees' learning performance and experience. The advent and rapid growth of internet technology and online tools support the offering of courses/programs affecting the frequency and manner in which interaction between the trainees and trainer is evident, provide and receive feedbacks on-time and the interaction with course materials with ease [3].

A significant paradigm in the delivery of education is the use of web-based systems/platforms. The Web as an education delivery medium provides information with excellent prospects and extend learning outside space and time boundaries [4]. With the Web, the conversion of traditional lectures and presentations into multimedia learning experiences for trainees is possible. With the availability of hardware and software, multimedia presentation is accessible that supports the use of colors, displays full-motion videos, and high acceptable audio [4]. Equally, resources like course materials; text; graphics and photographs; presentation programs; audio and video; animations; links to other websites or computer-based resources; e-mail; discussion forum; and tutorials enhance web-based learning as a medium for teaching and learning [5]. Subsequently, published guidelines for web learning are associated with collaboration, teacher and learner goals, motivation, communication, active learning, guidance and feedback, assessment, learner control, problem-solving, and authentic learning [6]. 
Alain C. Gallarde et al., International Journal of Emerging Trends in Engineering Research, 8(4), April 2020, 1148 - 1155

\subsection{CourseLab e-learning authoring system}

CourseLab is an e-learning authoring tool that quickly develops e-learning courses using its available templates and modules [7]. The design of CourseLab features heightens productivity in terms of content creation. It is also compliant with many e-learning standards such as AICC, SCORM 1.2, and 1.3. It is known due to its extensive set of ready-to-use e-learning module templates, at the same time supporting the What-You-See-Is-What-You-Get

(WYSIWYG) environment. Hence, complex HTML and other programming skills for content creations are not required. In terms of meeting the needs of different types of learners, the CourseLab provides different multimedia content such as graphics, texts, audio, and video, which are the features used to create assessment and evaluation of knowledge gathered from the lesson [8]. It also enhances the ability to self-learn, self-test, and self-report, thus matching the Competency-based Training (CBT) delivery. Furthermore, it offers the creation of tests that give instant feedback to the learners, a feat required for self-checks on the CBT. Thus, making CourseLab the ideal tool for the development of the e-CBT web App. However, there are new developments, new objectives, and ends that are redefining the development of technology and practice [9]. Henceforth, in providing quality education, the institution needs to implement an available platform, devising their e-learning addressing the needs of both trainers and trainees.

\subsection{Background of the Competency-Based Training (CBT) Program of TESDA}

The Southern Isabela College of Arts and Trades (SICAT) is a Technical Education Skills and Development Authority (TESDA) Training Institution offering Trade Technical Education Courses using Competency-Based Training (CBT) Program. Competency-Based Training (CBT) is a training delivery approach that focuses on the competency development of the trainee as a result of the training. It stresses what the trainee can perform; a timeframe is specified but focuses on outcomes rather than the learning process; and its primary goal is the realization and application of knowledge, skills, and attitude to a specific level of competency. The competency-based TVET (CBTVET) system introduces new learning methodologies and a unique learning environment where teachers are facilitators of learning. The CBTVET combined the self-learning approach and formal classroom instruction. The Competency-Based Learning Materials (CBLM) is a spectrum of educational materials that trainers use in the workshop to support specific learning outcomes on the CBT.

\subsection{Scope and Goal of the Study}

The proposed e-CBT framework design was applied in the development of the e-Competency-based training (e-CBT) web App for TESDA Technology Institutions (TTIs), focusing on the different modules and functionalities making use of the present CBT program as a basis. The conduct of testing activities in terms of the graphical user interface, network test, and compatibility ensures proper attainment of the App design and development. Moreover, the usability evaluation and the acceptability evaluation in terms of trainees' satisfaction, the learning experience of trainees, transfer of knowledge, skills, and attitudes, and the result of the CBT delivery warrants users' satisfaction and guarantees proper implementation and management of the App. The end-goal of the study serves as a basis for improving the overall results of the training. Notably, the school administrators to strategize budget allocation and improve efficiency in terms of purchasing and reproducing learning materials. Also, it offers real-time monitoring of trainees' learning progress and the automation of tasks such as discussion of trainees' and trainer's roles. More likely, the conduct of this study is in response to the Medium-Term Philippine Development Plan (MTPDP) 2004-2010 of TESDA.

\section{PROCEDURES AND METHODS}

\section{1. e-CBT Proposed Framework}

Figure 1 shows the proposed e-CBT (Competency-Based Training) framework applied in the development of the e-CBT web App. It covers the pre-assessment, selection of competencies, the learning module, institutional assessment, and the achievement chart. It intends for learning e-CBT on the Computer Systems Servicing NC II program/qualification. The National Certification (NC) II has the following competencies as stipulated in the TESDA Training Regulations: [a] Install and Configure Computer Systems, [b] Set-up Computer Networks, [c] Set-up Computer Servers, and [d] Maintain and Repair Computer Systems and Networks. The offering of the program/qualification is for 280 hours, taken for at least 2-3 months. The developed App guides trainers and trainees throughout the CBT delivery training approach from accommodation to exit of the program for trainees.

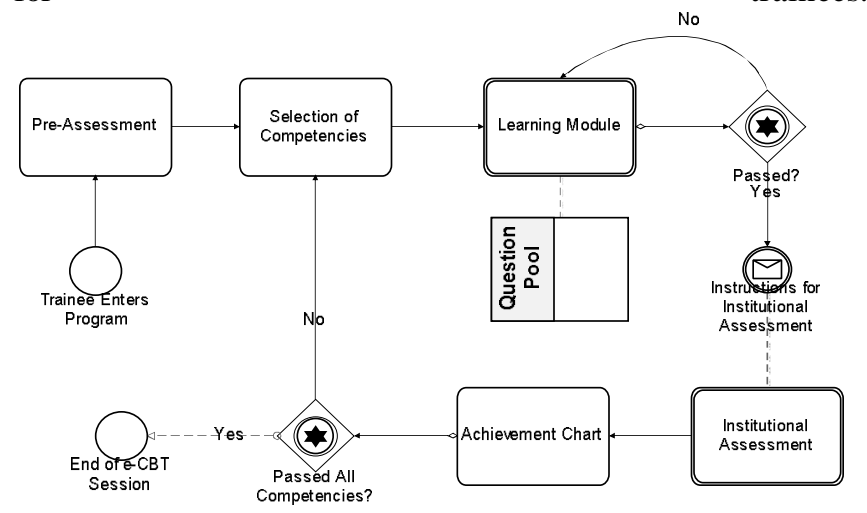

Figure 1: Proposed e-CBT Framework

Pre-Assessment. The pre-assessment module is similar to data gathering, where its primary goal is to assess the 
knowledge and skills of the trainees related to the CBT program through the administration, assessment, orientation, and dissemination of information. The identification of data like language, literacy, and numeracy was made possible with the use of the CBT data gathering instrument for Trainee's Characteristics. It is also capable of determining data such as cultural and language background, education and general knowledge, sex, age, physical ability, previous experience with the topic, prior learning experience, training level completed, specialized courses, learning styles, and other needs of the trainee. Afterward, the conduct of self-assessment checks which is essential in planning training sessions. It is a questionnaire determining trainees' capability of performing the Basic, Common, and Core competencies related to Computer Systems Servicing program/qualification. Based on their answers, the identification of training needs provides the right competencies, instructions, and training materials and resources.

Selection of Competencies. The trainer identifies and selects competencies based on trainee's pre-assessment results. The organization of learning strategies using a Session plan is available in the proposed framework. With this, the trainer can identify the learning contents, methods, presentation, practice, feedback, resources, and timeline for a particular competency.

Learning Module. The learning module includes the review of learning packages, usage of manuals, observing demonstration through the viewing of multimedia materials, practicing skills, and receiving assistance and advice. During the process, trainees practice and rates their performance, while trainer observes and rates performance in terms of demonstration and practicing skills. In the proposed framework, the web App automatically rates self-checks answered by the trainees.

Institutional Assessment. Institutional assessment is a practice test in preparation for the national assessment. The proposed framework includes an assessment at the end of every competency, where the App automatically rates the written exam and the evaluation of the overall performance based on demonstration skills.

Achievement Chart. An achievement chart is a monitoring tool used to check and evaluate the achievements of the trainees. Passing the Institutional Assessment triggers the achievement chart module updating the progress report of the trainees.

\subsection{Feasibility of the e-CBT Implementation}

The analysis of educational attainment in terms of computer literacy determines the feasibility of the e-CBT to trainees. As evidence, the training regulations (TR) of CSS NC II program/qualification state that "entry requirements of trainees must be at least high school graduate." Before the implementation of the Department of Education K-12 program, they embedded the usage of personal computers on their high school curriculum, specifically on Technology and Livelihood Education (TLE). Thus, the perception that trainees, whether high school graduates or higher, are capable of using the application that operates on personal computers.

\subsection{The Competency-Based Training (CBT) Process Flow}

To better understand the CBT process flow, trainees undergo pre-assessment determining their prior learning. The trainer set-up the CBT workshop into different workstations and components. The printed copies (paper-based) of Competency-Based Learning Materials (CBLM) are available and provided for the trainees. Before performing the required tasks, the trainees must read the information sheets and answer self-checks in the CBLM. Different monitoring instruments are set-up to monitor the trainee's progress and achievement. The trainees must pass the institutional assessment before moving to the next unit of competency competently. This approach heavily relies on printed copies, thus affecting the flow in case a problem occurs on these learning materials. Also, trainers are performing miscellaneous tasks such as checking of self-checks, task sheets, and job sheets have done manually. Figure. 2 presents the CBT process flow.

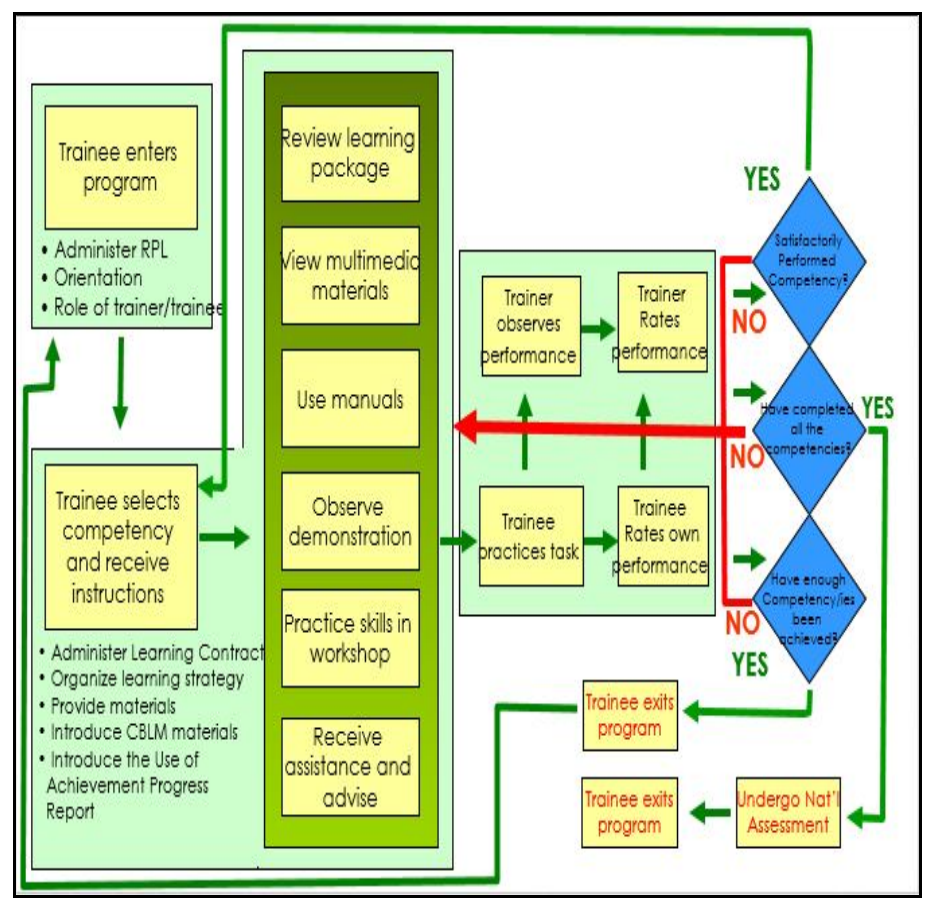

Figure 2: Competency-Based Training (CBT) Process Flow

\subsection{Infrastructure Layout of the e-CBT Web App}

The developed App is installed and maintained on the server-side of the network. The three-tier networks consist of three levels the client tier, application tier, and the database 
tier. The user interface layer that runs at the user's computer, a front-end layer, is the client-tier. The Google Chrome browser is the recommended browser. Meanwhile, the interaction between the client on one side and the database, referring to the database server and the content repository is the application-tier. The Apache Webserver runs in the Windows platform and PHP. A server-side scripting language to connect to the back-end (database). Lastly, the database server is the back-end layer that maintains the data needed for the web App. It stores information, including links to the content repository, and controls the necessary system functionality by using the MySQL database running in the Windows platform is the database-tier. Figure 3 presents the infrastructure layout of the e-CBT web App.

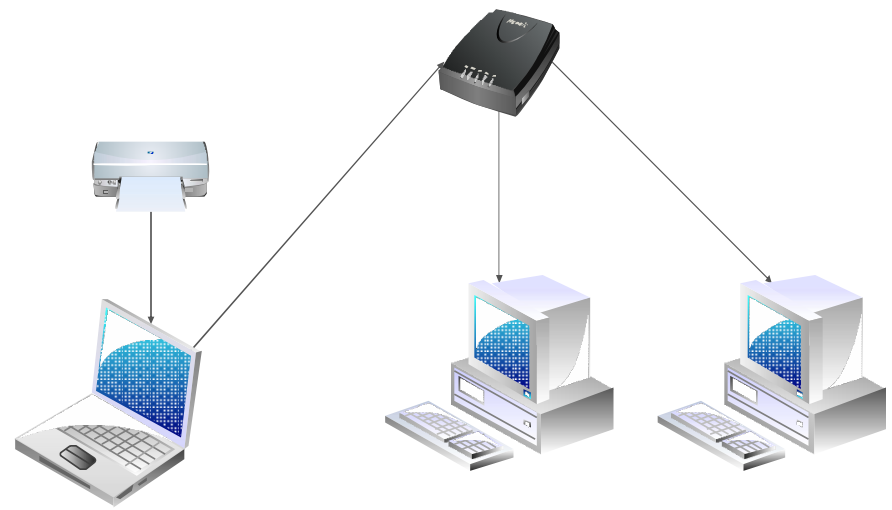

Figure 3: Infrastructure Layout of the e-CBT Web App

\subsection{Software Development Methodology}

Figure 4 shows the Rapid Application Development (RAD) model. The model includes four phases: (1) requirements analysis and quick design; (2) app development using the Prototype cycle of developing, demonstration, and refinement; (3) app testing; and (4) app evaluation.

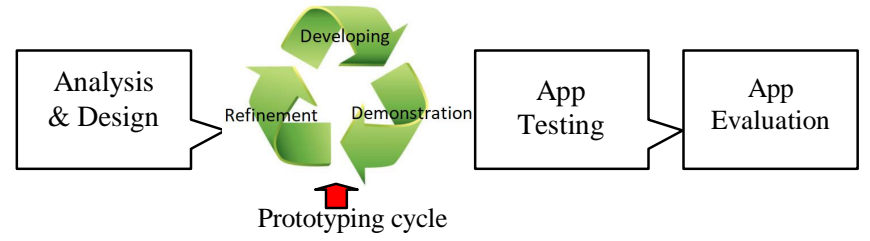

Figure 4: Rapid Application Development Model

\subsubsection{Requirements Analysis and Quick Design}

Based on the RAD model, requirements analysis determines the information provided by the end-users and functional requirements that guided the design and development of the web App. It also includes identifying the problems and limitations based on the standard Competency-Based Training delivery. The design phase includes interfaces, databases, and hardware architecture designs based on the requirements and functions. The use case diagram, as shown in Figure 5, shows what modules and processes the trainer and trainee can access and perform. Both can access modules related to pre-assessment, selection of competencies, learning modules, institutional assessment, and achievement chart. However, the registration of trainees is accessed only by the trainer since this module contains vital information about the trainees enrolled in the program.

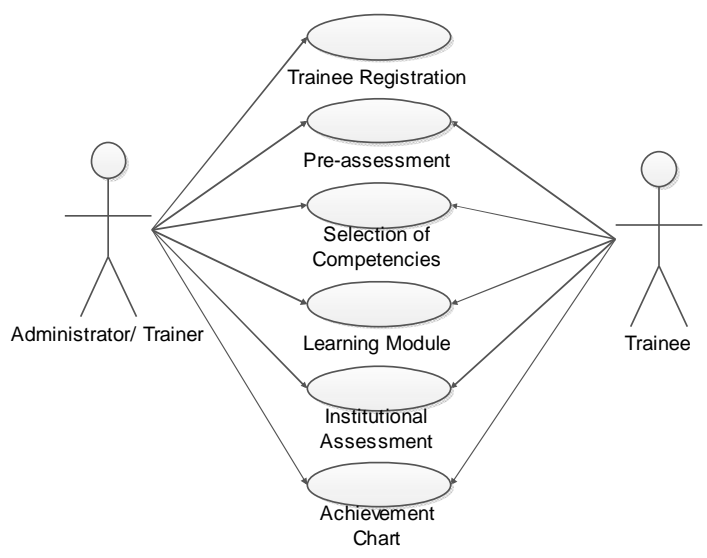

Figure 5: Use case diagram showing modules and processes a trainer and trainee can access and perform

The design of interfaces is compatible with a desktop computer ensuring more extensive display for essential information. All information that needs graphical representation, and multimedia materials such as video presentations, simulations, and photos were embedded to address visual learners. A sequence diagram shows the overall process of the App, and the administrator/trainer registers trainees on the e-CBT application before they can access the App. Trainees can now access the modules, as presented in Figure 6.

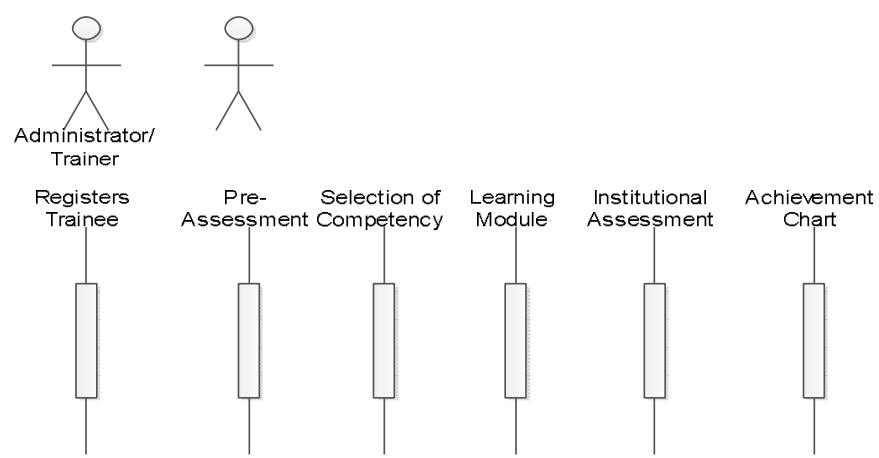

Figure 6: Sequence Diagram showing the overall process performed by the trainer and trainee

\subsubsection{Prototype cycles of developing, demonstration, and refinement}

Using the CourseLab e-learning authoring tool, the development of the e-CBT web App took placed. In the process, prototypes were created, demonstrated to technical experts, some peers, and trainees. Refinements follow after providing feedback for improvement. The Courselab had been used to create learning materials, task sheets, job sheets, self-checks, pre-assessment, and learning materials. Other software used includes Adobe Dreamweaver as the source 
code editor for the scripting language, the XAMPP for running the MySQL Server (Database), and the Apache Server for testing and modification of the components of the App. During the development, technologies like PHP, HTML conjunction with the CSS for design, achieving a user-friendly interface. JavaScript to run client-side functions, Apache for the webserver together with the MySQL because of its availability as open-source software.

\subsubsection{App Testing}

The Southern Isabela College of Arts and Trades (SICAT), a government institution offering TVET programs, serves as the testing and evaluation ground. During the testing phase, the graphical user interface test, network test, and compatibility testing were carried out.

\subsubsection{App Evaluation}

In the evaluation phase, three IT experts conducts an expert review to assess the usability of the e-CBT web App. The adoption of the usability heuristics by Jakob Nielsen with the following criteria: system status visibility; the match between system and real-world; user control and freedom; consistency and standards; prevention of errors; help users recognize, diagnose and recover from errors; and availability of help and documentation [10]. On the other hand, the Kirkpatrick model consists of four levels designed as a sequence of ways to evaluate training programs. The user's acceptability aspect of the e-CBT App in terms of trainee's satisfaction; learning experience of the trainee; transfer of knowledge, skills, and attitudes; and results of the CBT delivery using the evaluation model of Kirkpatrick, 2007 [11]. Before the trainees' ended the program/qualification, a questionnaire was prepared and floated for them to evaluate the e-CBT web App. The statements/questions are structured using the three-level Likert scale format. The choices represent the degree of agreement each trainee had on the given item with the highest detailed interpretation as "Very Satisfactory", "Satisfactory", and the lowest as "Poor".

\section{RESULTS AND DISCUSSIONS}

\subsection{Development of the e-CBT web app applying the proposed e-CBT framework}

The basis of developing the e-CBT web App is the proposed e-CBT framework. While using the App, the conduct of pre-assessment reduces time, effort, and providing convenience for both trainees and trainer. It also allows organizations and efficient trainees information profiling, as shown in Figure 7.

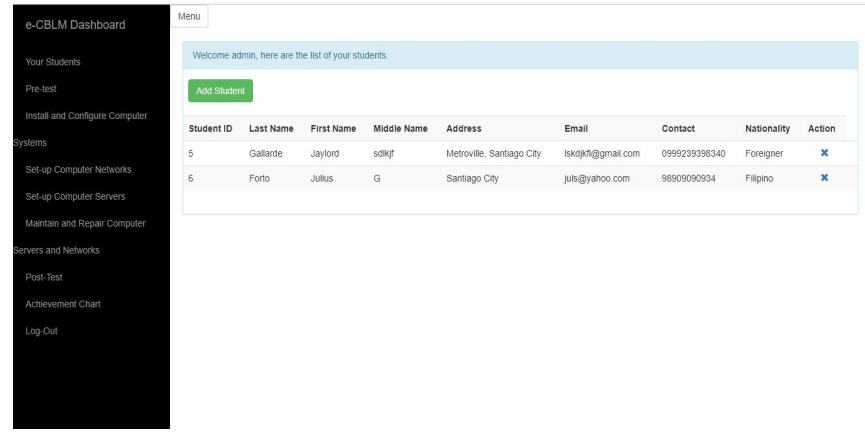

Figure 7: Student Profiling Module of the e-CBT

The web app provides a dashboard for Administrator/trainer to add, edit, and delete print, search trainees' information and the overall contents of the e-CBT App. To explain further, the Administrator Dashboard contains eight (8) main modules, namely:

1. Trainees' registration module allows the administrator/trainer to add, edit, delete, and print, search, and organize trainees' information profile.

2. The pre-test module enables trainees to take the pre-test to gauge their know-how before accessing the main course.

The e-CBT on Computer Systems Servicing NC II has the following core competencies shown on the dashboard

3. Install and Configure Computer Systems

4. Set-up Computer Networks

5. Set-up Computer Servers

6. Maintain and Repair Computer Systems and Networks

7. After finishing all the competencies, the conduct of the Post-test follows serving as an institutional assessment

8. Achievement Chart module allows trainer and trainees to monitor their overall progress throughout the training.

On the other hand, the Selection of Competencies shows competencies based on the result of training needs embedded in the App. The different options shown in Figure 8 includes:

1. The Introduction - shows the overall coverage of the competency program/qualification

2. Objectives - shows the general objective and specific objectives of the competency modules

3. Assessment Criteria - shows the criteria/rubric used for assessment/test

4. Learning Outcomes - indicates the learning deliverables attained for a specific competency

5. Competency Assessment - an institutional assessment perform at the end of each qualification 


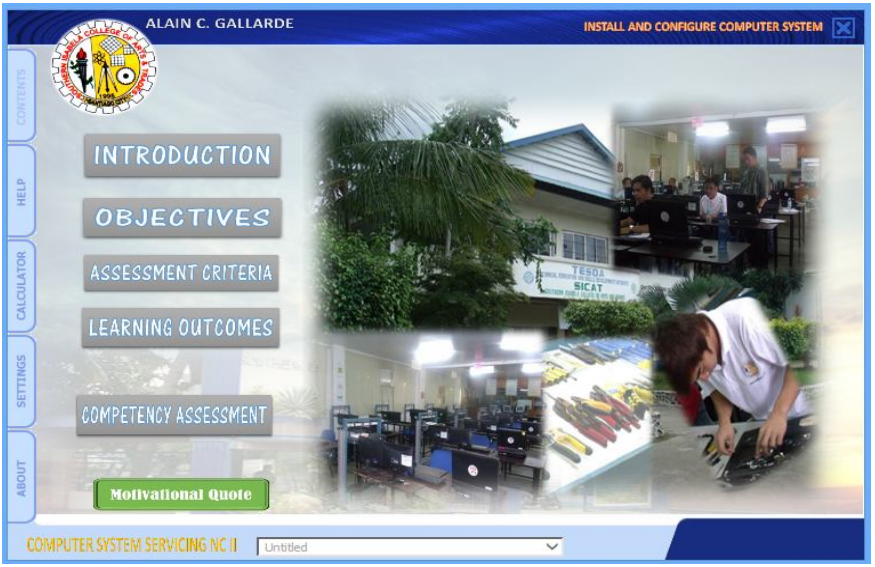

Figure 8: Selection of Competencies Module of the e-CBT

Afterward, if a specific trainee finishes a competency, the Achievement Chart is updated automatically indicating the progress of the trainee. And then the trainee can now select another module until he finishes all the competencies for a particular qualification.

\subsection{Testing the App}

In terms of App testing, the conduct of the graphical user interface test ensures that all users (trainees and trainer) of the e-CBT App can operate and navigate with ease, clarity, conciseness, familiarity, consistency, attractively, and efficiently. Another is the network test ensuring the App can be shared and accessed over the network. Lastly, compatibility testing ensures that various computers, ranging from desktop up to laptop, can access and navigate on the App with the same user experience.

\subsection{Evaluation of the Technical Aspects of the Web App}

An expert review considering three experts with experience in the field of e-learning evaluates the usability of the App. Table 1 presents the results of the evaluation.

Table 1: Experts' evaluation results in terms of usability

\begin{tabular}{|l|c|c|c|}
\hline Criteria & VS & S & P \\
\hline System status visibility & 3 & & \\
\hline Match between system and real-world & 2 & 1 & \\
\hline User control and freedom & 2 & 1 & \\
\hline Consistency and standards & 3 & & \\
\hline Prevention of errors & 3 & & \\
\hline $\begin{array}{l}\text { Helping users recognize, diagnose, and } \\
\text { recover from errors }\end{array}$ & 3 & & \\
\hline Availability of help and documentation & 3 & & \\
\hline
\end{tabular}

VS- Very Satisfactory; S- Satisfactory; P - Poor

In terms of system status visibility, the App provides notifications determining the state of the App in every operation a trainee may encounter. In consideration, the CBT manual process flow matches the App on a real-world basis.
The trainer and trainees have their control and freedom since they can choose their account preferences. In terms of consistency and standards, learning modules/contents are aligned on Competency Standards and Training Regulations set by TESDA. Try catch exception has been used on almost every code of the App to promote error prevention. The error notifications design is suggesting users what to do despite errors encountered. Lastly, the availability of help and documentation serves as references in terms of usage and troubleshooting. Overall, the technical experts highly commended the App.

In evaluating the App's acceptability, the Kirkpatrick's four levels of learning was adopted. There were three IT teachers, seventy (70) trainees, and ten (10) trainers serving as participants. The tabulation of answers and adding results determine the overall count of rating per criterion. The focus of the evaluation is on trainee's satisfaction, the learning experience, the transfer of knowledge skills and attitudes, and the result of the e-CBT delivery. The first is the reaction, which is represented by Trainee's Satisfaction. It is measured using assessing the experience of trainees in terms of navigation, images, colors, fonts, and overall acceptance of the application. The second level is learning, which is represented by the learning experience of trainees. It evaluates the implementation of RPL (Recognition of Prior Learning), orientation materials, the role of trainer and trainees, and other crucial phases on the e-CBT delivery. The third level is the behavior, represented by Transfer of Knowledge, Skills, and Attitudes. It involves self-checks and task sheets to determine the effectiveness of the transfer of knowledge. Lastly, the results, which aims to evaluate if the overall effect of the CBT delivery using the developed App, is favorable and acceptable to trainees.

As shown in Table 2, almost all criteria revealed that the trainees' satisfaction in terms of the navigation of the application, importance of pages, links, quality of custom images, and relatedness of colors garnered the highest rating of 83, with a descriptive equivalent of "Very Satisfactory". These results implied that trainees are highly satisfied with the App. On the other hand, consistency of font size garnered a rating of 80 with a descriptive equivalent of "Very Satisfactory", which revealed that standard and consistent font sizing is vital in delivering online materials/courses thus needing a little improvement. While rated Very Satisfactory, the lowest rating is on the consistency of font spacing, which garnered a score of 78 , this implied that font spacing is essential in the presentation of contents/modules of the App. Overall, the usage of the App provides a more convenient and productive approach in learning that is satisfactorily accepted by trainees. 
Alain C. Gallarde et al., International Journal of Emerging Trends in Engineering Research, 8(4), April 2020, 1148 - 1155

Table 2: Summary of overall rating per criterion under trainee's satisfaction

\begin{tabular}{|c|c|c|c|}
\hline \multirow[t]{2}{*}{ Trainee's satisfaction } & \multicolumn{3}{|c|}{$\begin{array}{c}\text { Trainees' } \\
\text { Rating }\end{array}$} \\
\hline & VS & $\mathbf{S}$ & $\mathbf{P}$ \\
\hline $\begin{array}{l}\text { 1. The navigation of the App is a clear } \\
\text { indicator of what your primary focus } \\
\text { and what you do best. }\end{array}$ & 83 & & \\
\hline $\begin{array}{l}\text { 2. The navigation was defined by first } \\
\text { understanding how users navigate } \\
\text { the App. }\end{array}$ & 83 & & \\
\hline $\begin{array}{l}\text { 3. The navigation methods and labels are } \\
\text { aligned with how you think and the } \\
\text { vernacular you use when talking } \\
\text { about learning outcomes. }\end{array}$ & 83 & & \\
\hline $\begin{array}{l}\text { 4. The most important pages of the App } \\
\text { are in the primary navigation. }\end{array}$ & 83 & & \\
\hline $\begin{array}{l}\text { 5. Links on the App are labeled with } \\
\text { anchor text that provides a clear } \\
\text { indication of where they lead. }\end{array}$ & 83 & & \\
\hline $\begin{array}{l}\text { 6. Custom images used in the App are of } \\
\text { consistent size and quality. }\end{array}$ & 83 & & \\
\hline $\begin{array}{l}\text { 7. Colors used in the App are } \\
\text { harmonically and logically related. }\end{array}$ & 83 & & \\
\hline $\begin{array}{l}\text { 8. The use of font sizes consistently for } \\
\text { each of the primary elements of the } \\
\text { page in the App. }\end{array}$ & 80 & 3 & \\
\hline $\begin{array}{l}\text { 9. Font spacing is consistent throughout } \\
\text { the App. }\end{array}$ & 78 & 5 & \\
\hline $\begin{array}{l}\text { 10. Overall, the usage of the App } \\
\text { provides a more convenient and } \\
\text { productive approach in learning that } \\
\text { is satisfactorily accepted by trainees. }\end{array}$ & 83 & & \\
\hline
\end{tabular}

VS- Very Satisfactory; S- Satisfactory; P - Poor

In terms of trainees' learning experiences, Table 3 shows that the orientation materials used in the App are current and accurate, garnering a perfect rating of 83, "Very Satisfactory". Alongside the discussion of the role of trainer and trainee, difficulty in selection of desired competencies, the accuracy of manuals presented in the App, administration of learning contract, and the overall trainee satisfaction with their learning experience. Meanwhile, providing proper administration of 'recognition of prior learning', embedment of multimedia materials, organization of learning strategies, and the CBLM attraction to trainees still got a "Very satisfactory" rating of 81. Overall, trainees are satisfied with their learning experiences.

Table 3: Summary of overall rating per criterion under the learning experience of trainees

\begin{tabular}{|c|c|c|c|}
\hline The learning experience of trainees & \multicolumn{3}{|c|}{$\begin{array}{c}\text { Trainees' } \\
\text { Rating }\end{array}$} \\
\hline & VS & S & P \\
\hline $\begin{array}{c}\text { 1. The App provides accurate } \\
\text { administration of RPL (Recognition }\end{array}$ & 82 & 1 & \\
\hline
\end{tabular}

\begin{tabular}{|c|c|c|}
\hline of Prior Learning). & & \\
\hline $\begin{array}{l}\text { 2. The orientation materials used in the } \\
\text { App are current and accurate }\end{array}$ & 83 & \\
\hline $\begin{array}{l}\text { 3. The role of the trainer and trainee is } \\
\text { available and clear in the App. }\end{array}$ & 83 & \\
\hline $\begin{array}{l}\text { 4. The selection of the desired } \\
\text { competency is not difficult. }\end{array}$ & 83 & \\
\hline $\begin{array}{l}\text { 5. Multimedia materials embedded in the } \\
\text { App are attractive to watch. }\end{array}$ & 82 & 1 \\
\hline $\begin{array}{l}\text { 6. Manuals presented in the App are } \\
\text { accurate and current. }\end{array}$ & 83 & \\
\hline $\begin{array}{l}\text { 7. The App has appropriately } \\
\text { administered the learning contract. }\end{array}$ & 83 & \\
\hline $\begin{array}{l}\text { 8. The learning strategy has been } \\
\text { organized correctly in the App. }\end{array}$ & 82 & 1 \\
\hline $\begin{array}{l}\text { 9. Competency-Based Learning } \\
\text { Materials (CBLM) attracts trainees } \\
\text { to study and learn more. }\end{array}$ & 82 & 1 \\
\hline $\begin{array}{l}\text { 10. Overall, trainees are satisfied with } \\
\text { their learning experience. }\end{array}$ & 83 & \\
\hline
\end{tabular}

Table 4 presents the results in terms of Transfer of Knowledge, Skills, and Attitudes. It shows that the usage of self-checks to ensure learning garnered the highest rating of 83, "Very Satisfactory". Alongside with the validation of transfer of knowledge using task sheets and usage of performance criteria checklist. On the other hand, the roles of trainers and trainees were meant to change the attitude of a trainee using following established house rules and other policies. The App accomplishes the transfer of knowledge, skills, and attitudes for trainees who got a rating of 80 , which needs little improvement.

Table 4: Summary of overall rating per criterion under Transfer of Knowledge, Skills, and Attitudes

\begin{tabular}{|c|c|c|c|}
\hline \multirow[t]{2}{*}{$\begin{array}{l}\text { Transfer of knowledge, skills, and } \\
\text { attitudes }\end{array}$} & \multicolumn{3}{|c|}{$\begin{array}{c}\text { Trainees' } \\
\text { Rating }\end{array}$} \\
\hline & VS & $\mathbf{S}$ & $\mathbf{P}$ \\
\hline $\begin{array}{l}\text { 1. Transfer of knowledge is ensured on } \\
\text { the App by providing self-checks to } \\
\text { ensure learning. }\end{array}$ & 83 & & \\
\hline $\begin{array}{l}\text { 2. The transfer of knowledge is validated } \\
\text { by providing a task sheet on the App. }\end{array}$ & 83 & & \\
\hline $\begin{array}{l}\text { 3. Task sheets accomplished by trainees } \\
\text { are evaluated on the App using the } \\
\text { performance criteria checklist } \\
\text { embedded on the App. }\end{array}$ & 83 & & \\
\hline $\begin{array}{l}\text { 4. The embedded roles of trainer and } \\
\text { trainee on the App changed the } \\
\text { attitude of the trainee by following } \\
\text { established house rules and other } \\
\text { policies. }\end{array}$ & 80 & 3 & \\
\hline $\begin{array}{l}\text { 5. Overall, the transfer of knowledge, } \\
\text { skills, and attitudes for trainees is } \\
\text { accomplished by the App. }\end{array}$ & 80 & 3 & \\
\hline
\end{tabular}


The results of the e-CBT delivery presented in Table 5 revealed that all criteria garnered equal ratings of 83, "Very Satisfactory". Hence, App utilization is acceptable for trainees.

Table 5: Summary of overall rating per criterion under Results of the e-CBT delivery using the App

\begin{tabular}{|c|c|c|c|}
\hline \multirow[t]{2}{*}{$\begin{array}{l}\text { Results of the e-CBT deliver using the } \\
\text { App }\end{array}$} & \multicolumn{3}{|c|}{$\begin{array}{l}\text { Trainees' } \\
\text { Rating }\end{array}$} \\
\hline & VS & $\mathbf{S}$ & $\mathbf{P}$ \\
\hline $\begin{array}{l}\text { 1. A trainee who has achieved a specific } \\
\text { qualification using the App is } \\
\text { competent in all related works. }\end{array}$ & 83 & & \\
\hline $\begin{array}{l}\text { 2. Trainers handled learning sessions } \\
\text { using the App can store essential } \\
\text { information such as accomplished } \\
\text { self-checks, data gathering } \\
\text { instruments, and monitoring tools for } \\
\text { program evaluation and future } \\
\text { planning. }\end{array}$ & 83 & & \\
\hline $\begin{array}{l}\text { 3. Overall, the result of the e-CBT } \\
\text { delivery using the App is favorable. }\end{array}$ & 83 & & \\
\hline
\end{tabular}

\section{CONCLUSION}

The proposed e-CBT framework derived the end-goal of improving the overall results of the competency-based training in learning Computer Systems Servicing (CSS) program/qualification. Also, the proposed framework aids in developing other e-CBT programs/qualificaton addressing the need for institutions of adopting an e-learning system to address and sustain TESDA's Medium-Term development plan. The developed e-CBT web app serves as an avenue for trainees to improve their knowledge, skills, and attitude, combining both the self-learning approach and formal classroom instruction. Thus, the web App as an education delivery medium provides current, organize, and accurate information and improves the competency of trainees extending learning outside classroom and time boundaries.

\section{ACKNOWLEDGEMENT}

The authors acknowledge the active participation of trainers, trainees, and administration management of Southern Isabela College of Arts and Trades (SICAT), Santiago City, and to the Isabela State University for the support of this kind of research work.

\section{REFERENCES}

[1] Christina Keller, Stefan Hrastinski, and Sven Carlsson, "Students' acceptance of e-learning environments: A comparative study in Sweden and Lithuania", Proceedings of the 15th European Conference on Information Systems, ECIS 2007, 395-406, 2007.
[2] Ali Tarhini, Kate Hone, Xiaohui Liu, and Takwa Tarhini, "Examining the moderating effect of individual-level cultural values on users' acceptance of E-learning in developing countries: a structural equation modeling of an extended technology acceptance model", Interactive Learning Environments. 10.1080/10494820.2015.1122635, 2016.

[3] A. Barman, R. Aziz, and Y. Yusoff, "Learning style Awareness and Academic Performance of Students", South East Asian Journal of Medical Education, 8(1), 2014. https://doi.org/10.4038/seajme.v8i1.124

[4] B.S. Sawant, and S.P. Shinde, "A Study of the effect of web-based education environments in schools: With special reference to Satara District", International Journal of Soft Computing and Engineering (IJSCE) ISSN: 2231-2307, Vol.1, 2012.

[5] A. Jolliffe, J. Ritter, and D. Stevens, The Online Learning Handbook: Developing and Using Web-based Learning. London: Kogan Page, 2001.

[6] S. Ssemugabi, and M.R. de Villiers, Usability and Learning: A Framework for Evaluation of Web-based e-Learning Applications, Research article, SACJ, No. 45, 2010. Available at http://sac j.cs.uct.ac.za/ index.php/ sacj/article/viewFile/37/20 https://doi.org/10.18489/sacj.v45i0.37

[7] Courselab Main, 2019. Available at http://www. courselab.com/view_doc.html?mode=home

[8] C.Y. Ragasa, "The CourseLab VLE: Its effect on the achievement and students' perception toward learning algebra", 21st Asian Technology Conference in Mathematics, Pattaya, Thailand, 2016.

[9] B. Fetaji, M. Fetali, and M. Ebib, "e-Learning systems comparative analyses", 5th International Conference on Informatics and Information Technology, 2016.

[10] Jakob Nielsen, Heuristics for User Interface Design, 1994. Available at https://www.nngroup.com/articles/ ten-usability-heuristics/ https://doi.org/10.1016/B978-0-08-052029-2.50008-5

[11] D.L. Kirkpatrick, Evaluating Training Programs: The Four Levels, 2nd Ed., San Francisco, Berrett-Koehler, 1998. 